\title{
A COMPACTNESS PROPERTY FOR PRIME IDEALS IN NOETHERIAN RINGS
}

\author{
CLIVE M. REIS AND T. M. VISWANATHAN
}

Abstract. A ring $R$ is compactly packed by prime ideals if whenever an ideal $I$ of $R$ is contained in the union of a family of prime ideals of $R, I$ is actually contained in one of the prime ideals of the family. It is shown that a commutative Noetherian ring is compactly packed if and only if every prime ideal is the radical of a principal ideal. For Dedekind domains this is equivalent to the torsion of the ideal class group and again to the existence of distinguished elements for the essential valuations. If a Noetherian ring $R$ is compactly packed then Krull $\operatorname{dim} . R \leqq 1$. Also a Krull domain $R$ is compactly packed if and only if it is a Dedekind domain with torsion ideal class group.

Throughout $R$ will be assumed to be a commutative ring with an identity element. The following property is well known $[1$, p. 8$]$ : if an ideal $I \subseteq \bigcup_{i=1}^{i=n} P_{i}$ where the $P_{i}$ are prime ideals of $R$ then $I \subseteq P_{i}$ for some $i$. This note discusses the situation when the union of any family of prime ideals of $R$ is considered.

We say that the ring $R$ is compactly packed (by prime ideals of $R$ ) if the following holds: $(*)$ if an ideal $I \subseteq \bigcup_{\alpha \in S} P_{\alpha}$ where the $P_{\alpha}(\alpha \in S)$ are prime ideals of $R$ then $I \subseteq P_{\alpha}$ for some $\alpha \in S$.

1. We shall first characterize those Noetherian rings which are compactly packed by prime irieals.

Theorem 1.1. A Noetherian ring $R$ is compactly packed by prime ideals of $R$ if and only if every prime ideal $P$ is the ratiical of a principal ideal $(r)$ in $R$ (or equivalently a positive power $P^{n} \subseteq(r)$ for some $r$ in $P$ ).

Proof. The condition is necessary. Let $P$ be a prime ideal in $R$ such that $P \neq \sqrt{ }(r)$ for any $r \in R$. Thus if $x \in P$ then $\sqrt{ }(x) \neq \neq P$. Let $P_{1}, P_{2}, \cdots, P_{k}(k \geqq 1)$ be the isolated prime components of the principal ideal $(x)$. Thus $\sqrt{ }(x)=P_{1} \cap P_{2} \cap \cdots \cap P_{k}[9$, Theorem 10, p. 213]. Since $x \in P$, one of the $P_{i}$ say $P_{1}$ is necessarily contained in $P$. We have two cases. Either $k=1$ in which case $P_{1 \neq}^{C} P$ or $k>1$. In that case again $P \subseteq P_{2}$, for from $P \subseteq P_{2}$, we will have $P_{1} \subseteq P \subseteq P_{2}$ contradicting the fact that $P_{1}, P_{2}$ are isolated prime components of $(x)$. Thus in either case, there exists a prime ideal $Q_{x}$ such that $P \Phi Q_{x}$ and 1969.

Received by the editors October 13, 1969 and, in revised form, November 22,

AMS Subject Classifications. Prinary 1315, 1325; Secondary 1398, 1270.

Key Words and Phrases. Noetherian ring, Dedekind domain, Krull domain, Krull dimension. 
$x$ belongs to $Q_{x}$. It follows that $P \subseteq \mathrm{U}_{x \in P} Q_{x}$. However $P$ is not contained in any $Q_{x}$ and so $R$ is not compactly packed.

The condition is sufficient. Observe that the condition implies that the radical $\sqrt{ } I$ of every ideal $I$ is equal to $\sqrt{ }(r)$ for some $r \in I$. Now if $I \subseteq \bigcup_{\alpha \in S} P_{\alpha}$ for prime ideals $P_{\alpha}$, then $\sqrt{ }(r)=\sqrt{ } I \subseteq \bigcup_{\alpha \in S} P_{\alpha}$. If $r \in P_{\beta}(\beta \in S)$ then $I \subseteq P_{\beta}$ and so $R$ is compactly packed.

Proposition 1.2. Let $R$ be a Noetherian ring in which every maximal ideal is the radical of a principal ideal. Then Krull dim. $R \leqq 1$.

Proof. By [9, Corollary 2, p. 239], the isolated prime components of any principal ideal $R a(\neq R)$ have height at most 1 . Hence the nonunits of $R$ are covered by prime ideals of height at most 1. In particular every maximal ideal $M$ of $R$ is contained in $U P_{\alpha}$ where the $P_{\alpha}$ are prime ideals of height at most 1. But $M=\sqrt{ }(r) \subseteq U P_{\alpha}$ and so from $r \in P_{\beta}$, we get $M \subseteq P_{\beta}$. Thus every maximal ideal of $R$ has height at most 1 . So Krull $\operatorname{dim} . R \leqq 1$.

Corollary 1.3. If a Noetherian ring $R$ is compactly packed then Krull dim. $R \leqq 1$.

REMARK 1.4. Observe that in view of Theorem 1.1, for Noetherian rings the defining condition $(*)$ for compactness can be replaced by the apparently weaker condition: (**) if a prime ideal $P \subseteq \bigcup_{\alpha \in S} P_{\alpha}$ of prime ideals $P_{\alpha}$ of $R$, then $P \subseteq P_{\alpha}$ for some $\alpha \in S$. Again Proposition 1.2 shows that a Noetherian ring $R$ is compactly packed if and only if the following condition holds: every maximal ideal of $R$ as well as every prime ideal of height 0 is the radical of a principal ideal. The question arises whether the second requirement above can be dropped even for Noetherian nondomains. The authors are unable to resolve this. However it is interesting to remark that a Noetherian semilocal ring of Krull dimension $\leqq 1$ is always compactly packed and so for such a ring, given an ideal $I$, there exists $r$ in $R$ with $\sqrt{ } I=\sqrt{ }(r)$.

2. We shall now present an interesting point of view on Dedekind domains. The following result is easily verified.

Proposition 2.1. Let $R$ be a ring and $M$ a multiplicatively closed subset of $A$. If $R$ is compactly packed, so is $R_{M}$.

Let $R$ be a Dedekind domain and $\rho$ the family of all proper prime ideals of $R$. If $P \in P$ then $R_{P}$ is a rank 1 discrete valuation ring and $R=\bigcap_{P \in \mathcal{Q}} R_{P}$. If $R^{\prime}$ is a domain such that $R \subseteq R^{\prime} \neq K$ where $K$ is the quotient field of $R$, then $R^{\prime}=\bigcap_{P \in Q} R_{P}$ for the subfamily $\mathcal{Q}$ of prime ideals $P \in \mathcal{Q}$ such that $R^{\prime} \subseteq R_{P}$. Also $R^{\prime}$ is a Dedekind domain [4. 
Propositions 1.1 and 1.2 , p. 799]. We have the following characterization of compactly packed Dedekind domains.

Theorem 2.2. Let $R$ be a Dedekind domain and $K$ its quotient field. Then the following statements are equivalent:

(1) $R$ is compactly packed by its prime ideals.

(2) The ideal class group of $R$ is torsion.

(3) Every domain $R^{\prime}$ such that $R \subseteq R^{\prime} \subseteq K$ is a ring of quotients of the form $R^{\prime}=R_{M}$ for some multiplicatively closed subset $M$ of $R$.

(4) If $\left\{v_{P}: P \in \mathcal{P}\right\}$ is the family of essential valuations of $R$ then for each $P \in P$ there exists $x_{p} \in R$ such that $v_{P}\left(x_{p}\right)>0$ and $v_{W}\left(x_{p}\right)=0$ for every $W \neq P$ in $P$. (Compare a corresponding result for Krull domains $[10$, Theorem 27, p. 83].)

Proof. By Theorem 1.1, (1) is equivalent to (2) if we remark that in a Dedekind domain the prime ideal $P$ is the radical of an ideal $I$ if and only if $I=P^{n}(n \geqq 1)$. That (2)↔(3) follows from [3, Proposition 1 , p. 219] and the introduction to this section. (2) $\Rightarrow(4)$. For if $P^{n}=(a)$ for some $n \geqq 1$, then $v_{P}(a)>0$ and $v_{W}(a)=0$ for every $W \neq P$ in $\odot$. Finally $(4) \Rightarrow(1) . \quad P \subseteq \bigcup_{W \neq P} P_{W}$ is impossible as $x_{p} \notin P_{W}$ if $W \neq P$ while $x_{p} \in P$.

Corollary 2.3. If $R$ is a compactly packed Dedekind domain with quotient field $K$ and $R \subseteq R^{\prime} \subseteq K$ then $R^{\prime}$ is also a compactly packed Dedekind domain.

COROLLARY 2.4. The ring of algebraic integers of an algebriac number field is a compactly packed Dedekind domain.

3. We shall now turn our attention to Krull domains.

THEOREM 3.1. A Krull domain $R$ is compactly packed if and only if it is a Dedekind domain with torsion ideal class group.

Proof. Let $R$ be a Krull domain. Then $R=\bigcap_{v \in \mathcal{F}} R_{v}$ where $\mathcal{F}$ is the family of essential valuations of $R$ and $R_{v}$ are the corresponding valuation rings. If $0 \neq x$ in $R$, then $v(x)=0$ for every $v \in \mathcal{F}$ will give $v(1 / x)$ $=0$ for every $v \in \mathcal{F}$ so that $1 / x$ belongs to $R$ and $x$ will be a unit in $R$. Thus for every nonunit $x$ in $R$, there exists $v_{x} \in \mathcal{F}$ with $v_{x}(x)>0$. So $x$ belongs to the centre $P_{x}$ of $v_{x}$. Thus the nonunits of $R$ are covered by all the minimal prime ideals of $R$. If $R$ were compactly packed, the last statement implies that every proper prime ideal in $R$ is a minimal prime ideal. [10, Theorem 28, p. 84] shows that $R$ is a Dedekind domain. Theorem 2.2 completes the proof. 
Corollary 3.2. A UFD is compactly packed if and only if it is a PID.

Corollary 3.3. An integrally closed Noetherian domain is compactly packed if and only if it is a Dedekind domain with torsion ideal class group.

REMARK 3.4. The proof of Theorem 3.1 also shows that in a Krull domain $R$ compactness with respect to minimal prime ideals (namely, if an ideal $I \subseteq \bigcup_{\alpha \in S} P_{\alpha}$ where the $P_{\alpha}$ are minimal prime ideals in $R$ then $I \subseteq P_{\alpha}$ for some $\alpha \in S$ ) is equivalent to compactness with respect to all prime ideals.

Acknowledgement. This paper is an extension and complete revision of a note originally submitted. The authors are grateful to the referee for his encouragement and interest.

\section{REFERENCES}

1. M. F. Atiyah and I. G. Macdonald, Introduction to commutative algebra, AddisonWesley, Reading, Mass., 1969.

2. N. Bourbaki, Algèbre commutative. Chapitre 7: Diviseurs, Hermann, Paris, 1965.

3. L. Claborn, Every abelian group is a class group, Pacific J. Math. 18 (1966), 219-222. MR 33 \#4085.

4. - Dedekind domains: Overrings and semi-prime elements, Pacific J. Math. 15 (1965), 799-804. MR 32 \#5682.

5. - Dedekind domains and rings of quotients, Pacific J. Math. 15 (1965), 5964. MR $31 \# 2263$.

6. H. Pollard, The theory of algebraic numbers, Carus Monograph Series no. 9, The Mathematical Association of America, Buffalo, New York, 1950. MR 12, 243.

7. P. Ribenboim, Theory of valuations, Lecture Notes, Queen's University, Kingston, 1967.

8. P. Samuel, Progrès recénts d'algèbre locale, Notas Math., no. 19, Instituto de Matemática Pura e Aplicada de Conselho Nacionale de Pesquisas, Rio de Janeiro, 1959. MR $26 \# 2458$.

9. O. Zariski and P. Samuel, Commutative algebra. Vol. I, The University Series in Higher Mathematics, Van Nostrand, Princeton, N. J., 1958. MR 19, 833.

10. - Commutative algebra. Vol. II, The University Series in Higher Mathematics, Van Nostrand, Princeton, N. J., 1960. MR 22 \#11006.

University of Western Ontario, London, Ontario, Canada 